\title{
Strong Optomechanical Squeezing of Light
}

\author{
T. P. Purdy, ${ }^{*}$ P.-L. Yu, R. W. Peterson, N. S. Kampel, and C. A. Regal \\ JILA, University of Colorado and National Institute of Standards and Technology, \\ and Department of Physics, University of Colorado, Boulder, Colorado 80309, USA
}

(Received 16 June 2013; revised manuscript received 12 August 2013; published 3 September 2013)

\begin{abstract}
We create squeezed light by exploiting the quantum nature of the mechanical interaction between laser light and a membrane mechanical resonator embedded in an optical cavity. The radiation-pressure shot noise (fluctuating optical force from quantum laser amplitude noise) induces resonator motion well above that of thermally driven motion. This motion imprints a phase shift on the laser light, hence correlating the amplitude and phase noise, a consequence of which is optical squeezing. We experimentally demonstrate strong and continuous optomechanical squeezing of $1.7 \pm 0.2 \mathrm{~dB}$ below the shot-noise level. The peak level of squeezing measured near the mechanical resonance is well described by a model whose parameters are independently calibrated and that includes thermal motion of the membrane with no other classical noise sources.
\end{abstract}

DOI: 10.1103/PhysRevX.3.031012

Interferometry is a ubiquitous method for sensitive displacement measurements. In typical interferometry employing a coherent state, the amplitude and phase quantum fluctuations are both at the shot-noise level. Recently, optomechanical systems have been developed that not only measure mechanical motion but can also manipulate the motion with radiation pressure. For example, radiation forces have been used to cool mechanical resonators to near their quantum ground state $[1,2]$. With sufficiently strong radiation pressure, quantum fluctuations can become the dominant mechanical driving force, leading to correlations between the mechanical motion and the quantum fluctuations of the optical field [3]. Such correlations can be used to suppress fluctuations on an interferometer's output optical field below the shot-noise level $[4,5]$, at the expense of increasing fluctuations in an orthogonal quadrature. This optomechanical method of manipulating the quantum fluctuations has historically been termed ponderomotive [6] squeezing.

The history of optical squeezing is intimately linked to quantum-limited displacement sensing [7], owing to proposals to increase the displacement sensitivity of largescale gravitational-wave observatories with squeezed light [8-11]. Squeezed light was first produced using atomic sodium as a nonlinear medium [12] and was soon followed with experiments employing optical fibers [13] and nonlinear crystals [14]. Substantial squeezing has been achieved in modern experiments (up to $12.7 \mathrm{~dB}$ [15]), and enhanced sensitivity using squeezed light has been realized in gravitational-wave detectors [16] and in biological

\footnotetext{
*tpp@jila.colorado.edu
}

Published by the American Physical Society under the terms of the Creative Commons Attribution 3.0 License. Further distribution of this work must maintain attribution to the author(s) and the published article's title, journal citation, and DOI.
Subject Areas: Optics, Quantum Physics

measurements [17]. Squeezed microwave fields, which have now been demonstrated with up to $10 \mathrm{~dB}$ of noise suppression [18], are an important tool in quantum information processing with superconducting circuits.

Early on, searches for ever-better squeezing materials led to suggestions that an optomechanical cavity, in which radiation pressure proportional to optical intensity changes the cavity length, could act as a low-noise Kerr nonlinear medium $[4,5,19]$, and hence could be a useful source of squeezed light [20]. Further, a unique advantage of utilizing an optomechanical nonlinearity is that correlations induced by a mechanical object can be used to enhance displacement sensitivity for that same object $[11,21]$.

However, experimentally, it has proven difficult to realize the substantial interplay between mechanical motion and quantum fluctuations of light required for ponderomotive squeezing. Early on, radiation-pressure-induced optical nonlinearity (bistability) was experimentally demonstrated in a cavity with a pendulum-suspended end mirror [22]. More recently, ponderomotively squeezed light at the few-percent level has been demonstrated using a mechanical mode of an ultracold atomic gas inside an optical cavity [23], and very recently using a silicon micromechanical resonator [24]. The former experiment was limited by nonlinearities in the interaction and the latter by excess mechanical thermal motion. Here, we observe ponderomotive squeezing at $1.7 \pm 0.2 \mathrm{~dB}$ below (32\% below) the shot-noise level and optical amplification of quantum fluctuations by over $25 \mathrm{~dB}$. The squeezing is realized on light transmitted through a Fabry-Perot optical cavity with an embedded, mechanically compliant dielectric membrane.

An optomechanical system can be thought of as an effective Kerr medium, and hence ponderomotive squeezing can be understood using many of the same ideas as typical nonlinear media. However, in ponderomotive squeezing, the finite mechanical response time defined by 
a complex mechanical susceptibility $\chi_{m}(\omega)$ plays an important role [19]. We can illustrate the features of our expected ponderomotive squeezing by tracing the quantum fluctuations $\delta X_{I}$ and $\delta X_{Q}$ in the optical amplitude and phase quadratures, respectively, propagating through our optomechanical cavity (Fig. 1). A large coherent state, referred to as the signal beam, consisting of nearly monochromatic radiation at a frequency $\omega_{L}$ and vacuum fluctuations at all other frequencies, enters the cavity from the left, and a vacuum state enters from the right. We first consider the simplest case where the laser-cavity detuning $\Delta$ is zero. Because the membrane is located in a spatial gradient of the standing-wave optical intensity, it is subject to an optical force from the shot-noise intensity fluctuations, termed radiation-pressure shot noise (RPSN). The membrane responds to the RPSN drive with motion concentrated at frequencies near its mechanical resonances, i.e., weighted by $\chi_{m}(\omega)$. The mechanical motion of the dielectric membrane causes fluctuations in the cavity resonance frequency that are imprinted onto the optical phase quadrature, yielding $\delta X_{Q}(\omega) \rightarrow \delta X_{Q}(\omega)+r(\omega) \delta X_{I}(\omega)$, while $\delta X_{I}$ remains unchanged. Here, $r(\omega)$ is a dimensionless complex Kerr parameter proportional to the strength of the coupling, which depends upon a variety of parameters, including the mechanical response. This Kerr-like self-phase-modulation correlates the amplitude and phase quadratures. If $r$ is real, the correlations destructively interfere in some particular quadrature, leading to squeezing, as illustrated in Fig. 1(c). If $r$ is purely imaginary, the added phase fluctuations do not lead to squeezing. This case occurs when probing on the optical resonance and measuring at the mechanical resonance frequency, where the mechanical response is perfectly out of phase with the RPSN drive.

Another consequence of the finite response time of the mechanical element [i.e., the imaginary component of $\left.\chi_{m}(\omega)\right]$ is that our system is directly coupled to the thermal bath. Thermal motion of the membrane imprints excess noise onto the light, which is uncorrelated with the optical shot noise and hence can limit the level of squeezing. To obtain significant squeezing near the mechanical resonance, the level of RPSN relative to the thermal force driving the membrane should be large [3]. For a beam with laser-cavity detuning near zero, this ratio is given by $R=C / n_{\text {th }} \times$ $\left[1+\left(2 \omega_{m} / \kappa\right)^{2}\right]^{-1}$, where $C=4 \bar{N} g^{2} / \kappa \Gamma_{0}$ is the optomechanical cooperativity, $\bar{N}$ is the average intracavity photon occupation, $g$ is the optomechanical coupling rate, $\Gamma_{0}$ is the mechanical dissipation rate, $\kappa$ is the cavity decay rate, $\omega_{m}$ is the mechanical resonance frequency, and $n_{\text {th }}$ is the thermal occupation of the mechanical state.

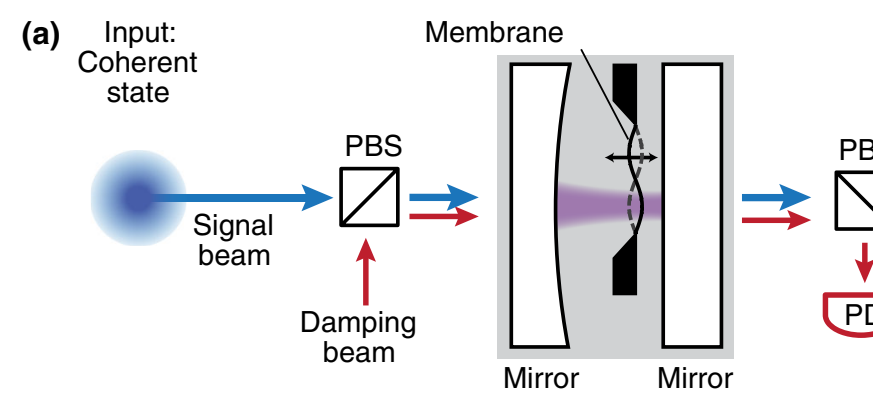

(b)

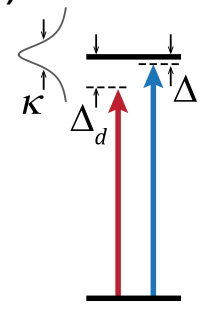

(c)

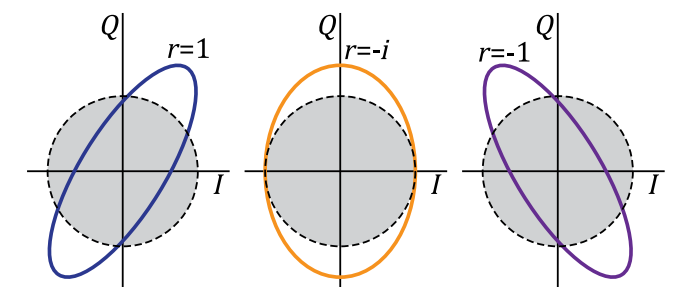

Output:

Squeezed state

\section{.}


Figure 1(d) illustrates the basic features we expect to see when measuring the squeezing in homodyne detection as a function of quadrature angle $\phi$ and detuning with respect to the mechanical resonance. To create this map, we use the Heisenberg-Langevin model described in the Appendix that captures more of the complexity of our system. In the diagram, we can see that for pure intensity quadrature light, we do not observe squeezing. However, as one rotates toward the phase quadrature, squeezing appears. The line shape of the squeezing is not symmetric about $\omega_{m}$ in our case but instead follows a Fano-like line shape. The vacuum fluctuations directly reflected off the output mirror interfere with the quadrature-rotated, mechanicalresonance-modulated light exiting the cavity. This diagram also illustrates the basic role of the mechanical susceptibility that weights the interaction between the membrane and the light. Namely, the magnitude of the squeezing $\left(S_{\phi}<1\right)$ and the antisqueezing $\left(S_{\phi}>1\right)$ fall off on the scale of the mechanical linewidth.

In our experiments, we also operate with a finite lasercavity detuning. At a finite $\Delta$, an understanding of the spectrum of fluctuations must also take into account the $\Delta$-dependent quadrature rotation of the intracavity states relative to the input fields $\phi_{c}=\tan ^{-1}(2 \Delta / \kappa)$ (i.e., offresonant phase fluctuations are partially converted into intracavity amplitude fluctuations and vice versa). This quadrature rotation generates squeezing in the amplitude quadrature, which may be observed via direct photodetection in addition to homodyne detection. The spectral line shape is also altered by the optomechanical damping and spring effects of the signal beam [25] on the membrane's mechanical response (see the Appendix).

Our optomechanical cavity (see Fig. 1 and Ref. [26]) consists of a 40-nm-thick by $500-\mu \mathrm{m}$-square silicon nitride membrane inside a 3.54-mm-long Fabry-Perot optical cavity [27]. We work with the $(2,2)$ drumhead mode of the membrane, with two antinodes along each transverse direction, yielding a mechanical resonance frequency of $\omega_{m}=2 \pi \times 1.524 \mathrm{MHz}$, and mechanical dissipation rate $\Gamma_{0}=2 \pi \times 0.22 \mathrm{~Hz}$. The interaction Hamiltonian $\hbar g N z$, where $z$ is the operator of the effective mechanical coordinate and $N$ is the intracavity photon-number operator, is equivalent to that of a harmonically bound end-mirror optomechanical cavity [4,5]. In our system, $g=$ $2 \pi \times 33 \mathrm{~Hz}, \kappa=2 \pi \times 1.7 \mathrm{MHz}$, and in a helium flow cryostat with a base temperature of $4.6 \mathrm{~K}$, we achieve $R>5$, when operating with $\bar{N} \sim 10^{8}$. This ratio is much larger than achieved in previous work [3], mainly because of increased optomechanical coupling. In addition to our main signal beam, we inject another laser into the orthogonal polarization cavity mode. This damping beam has a much weaker power than the signal beam but is detuned by $\Delta_{d} \sim-\omega_{m}$ from the cavity resonance. The damping allows us to avoid parametric instability and work with a mechanical mode with an effective mode temperature of less than $1 \mathrm{mK}$. See the Supplemental Material for more details about experimental methods and calibrations [28].

In our first set of experiments, we use direct photodetection to measure the power spectrum of the amplitude quadrature $S_{I}(\omega)$, which is normalized such that the detected shot noise is unity. Figure 2(a) shows $S_{I}$ for several values of $\Delta$, all at an average transmitted signal-beam power of $110 \mu \mathrm{W}$ corresponding to $\bar{N}=1.1 \times 10^{8}$ or $R=5.1$. A dip in noise below the shot-noise level is visible in the vicinity of $\omega_{m}$, a clear signature of squeezed light. The squeezing becomes more pronounced as $|\Delta|$ is increased because the maximally squeezed quadrature is rotated toward the amplitude quadrature. The data show excellent agreement over most of its frequency range with a Heisenberg-Langevin model, including quantum-noiselimited input optical fields, a thermally occupied mechanical bath coupled to the membrane, and no other classical noise sources (see the Appendix). However, a small excess of classical noise is visible at the largest detuning, a few tens of $\mathrm{kHz}$ above the mechanical resonance. Here, cavity frequency noise induced from a thermally occupied mechanical mode of the optomechanical cavity support structure [26] is increasingly converted in amplitude noise at larger $|\Delta|$. All of the system parameters used to generate the theory curves of Fig. 2(a) are independently measured, except $\Delta$ is calibrated, in part, using the displayed data.

The shot-noise level for the data of Fig. 2 is calibrated using balanced direct detection. The transmitted signal is split into two equal power beams and directed onto a pair of nearly identical photodetectors. Taking the sum of the detected signals is equivalent to single-detector direct detection. However, taking the difference of the detected signals removes classical and quantum correlations, up to the $20-\mathrm{dB}$ achieved common mode suppression. The difference signal consists of only the uncorrelated shot-noise level and a small, approximately 5\% contribution from the photodetector dark noise. $S_{I}$ is computed by taking the ratio of the power spectra of the sum and difference signals, after subtracting the measured photodetector dark noise.

The limits of the detected squeezing are illustrated in Fig. 2(b), where the minimum measured value of $S_{I}$ is plotted as a function of $\Delta$. The squeezing is limited somewhat by the thermal-noise-to-RPSN ratio $1 / R$. The finite quantum efficiency of our detection system is the largest limit to the detected squeezing. Including losses associated with the cavity $\epsilon_{c}=0.6$, propagation to the photodetector $\epsilon_{p}=0.8$, and photodetector conversion efficiency $\epsilon_{d}=$ 0.87 , we estimate an overall quantum efficiency of $\epsilon=$ $\epsilon_{c} \epsilon_{p} \epsilon_{d}=0.42$.

For Fig. 2(c), white classical intensity noise with an amplitude much greater than shot noise has been introduced onto the signal beam prior to entering the cavity. While classical intensity noise is clearly suppressed as well, the line shape of $S_{I}$ is qualitatively different from that of the quantum noise case. In the classical noise case, 

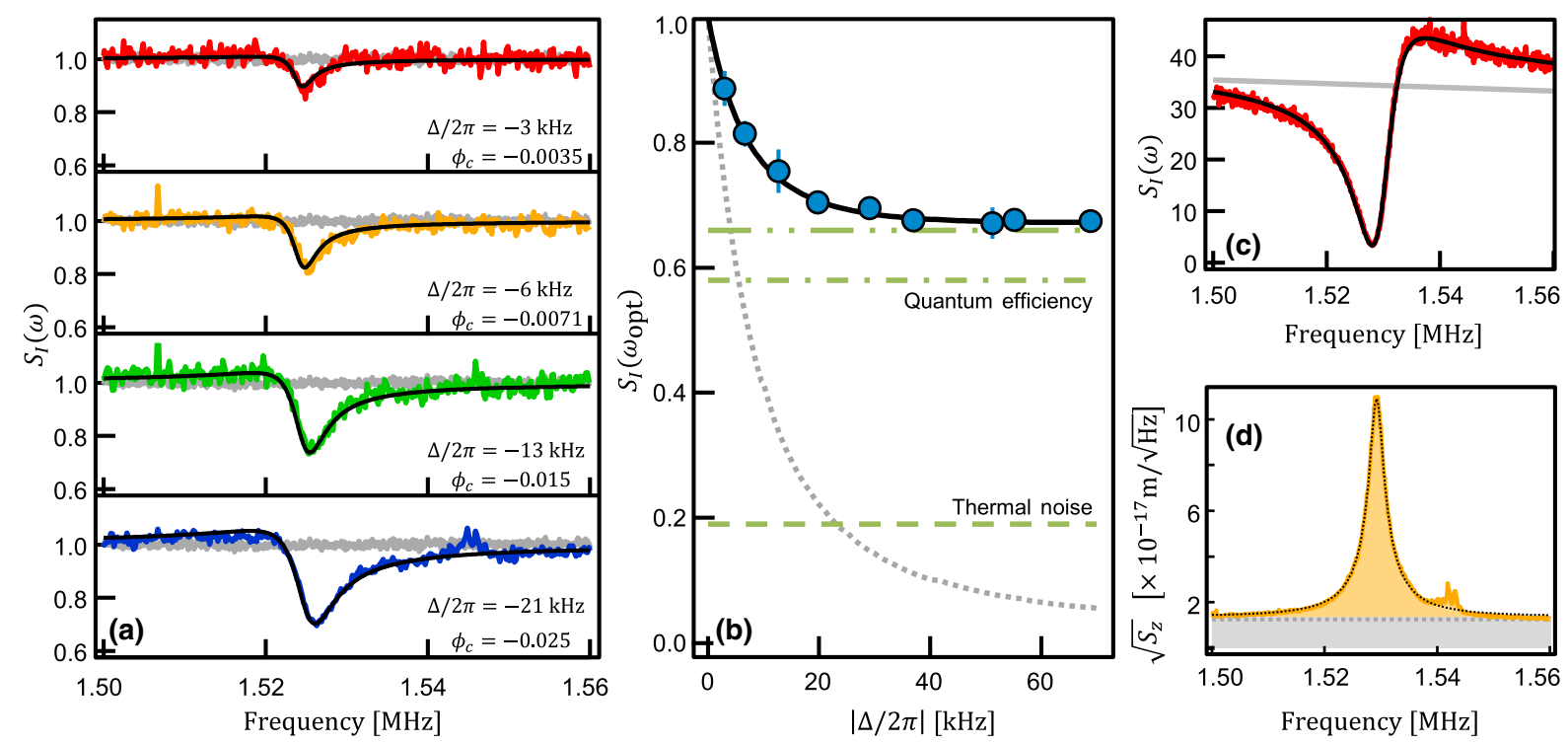

FIG. 2. Quantum intensity noise suppression. (a) Directly detected optical intensity noise signal-beam spectra for several signalbeam detunings. Also displayed are the measured shot-noise level (gray curves) and the theoretical predictions (black curves). A $200-\mathrm{Hz}$ bandwidth is used. The ratio of RPSN relative to thermal drive $R$ is fixed at 5.1. The damping beam provides $\Gamma_{0}^{\text {eff }} / 2 \pi=$ $2.7 \mathrm{kHz}$ and $\omega_{m}^{\text {eff }} / 2 \pi=1.524 \mathrm{MHz}$. However, the total mechanical damping rate and resonance frequency change with the signalbeam detuning $\Delta$. (b) The minimum value of $S_{I}$ for spectra as displayed in (a) (blue circles). Statistical error bars indicate the standard deviation. The frequency where the minimum occurs $\omega_{\text {opt }}$ shifts with $\Delta$ because of the optical spring effect. Also displayed are the mechanical thermal noise floor for our current parameters $1 / R$ (dashed green line), the limit set by finite detection efficiency $1-\epsilon$ (dot-dashed green line), the sum of the thermal and detection efficiency limits (dot-dot-dashed green line), and expected squeezing in the absence of optical loss and thermal motion (dotted gray line). (c) The directly detected optical intensity noise signal-beam spectrum with intentionally added white, classical amplitude noise (red curve), theoretical prediction (black curve), and level of added amplitude noise (gray line). (d) The mechanical displacement spectrum inferred from the damping-beam transmission spectrum (orange region) and Lorentzian fit (dotted black line). The detection noise floor is also shown (dotted gray line). One additional peak due to excess noise is visible in the bottom panel of (a) and in (d) at frequencies of approximately $1.545 \mathrm{MHz}$ due to a thermally occupied mechanical mode of the cavity support structure.

the Fano asymmetry reverses because of the absence of coherent interference, with phase and amplitude noise directly reflected from the output mirror, in contrast to the quantum noise case. A Heisenberg-Langevin model incorporating the additional classical laser noise term agrees well with the measured data. This symmetry difference between classical and quantum noise provides added confirmation that the spectra of Fig. 2(a) truly arise from the manipulation of quantum noise.

Although the transmitted signal-beam intensity spectrum is decidedly non-Lorentzian, the mechanical displacement still follows a simple Lorentzian form. The damping-beam transmitted intensity spectrum acts as a probe of mechanical motion uncomplicated by strong quantum correlations because its intensity and thus RPSN effects on the membrane are small [3]. The mechanical displacement spectrum derived from the damping beam [Fig. 2(d)] shows that the mechanics still retains a Lorentzian response to locally white force fluctuations.

We next explore all quadratures of the transmitted signal beam with balanced homodyne detection. We interfere the transmitted signal beam with an optical local oscillator whose phase is stabilized relative to the signal beam (see the Supplemental Material for details [28]). In Fig. 3(a), optical quadrature spectra $S_{\phi}(\omega)$ over a varying quadrature phase $\phi$ are displayed. $S_{\phi}$ is normalized such that the measured shot-noise level is unity. The phase $\phi=0$ corresponds to the amplitude quadrature and gives information equivalent to that obtained in the direct detection discussed above. (Note that for these measurements, $\Delta=$ $-2 \pi \times 42 \mathrm{kHz}$, allowing some squeezing to be rotated into the amplitude quadrature.) Regions with noise spectral density below the shot-noise level are visible over a bandwidth of approximately $100 \mathrm{kHz}$ and $\phi$ ranging over tens of degrees. The range of observed squeezing is limited to a region smaller than predicted by theory because of the thermal motion of the cavity mirrors and support structure mentioned above. The depth of the observed squeezing in homodyne detection is also lower than that observed in direct detection. This reduction is partially accounted for by imperfect overlap between the probe beam and homodyne local oscillator, contributing an additional effective optical loss $\epsilon_{m}=0.8$. Also, we operate in a regime where the homodyne local oscillator power is only a factor of less 


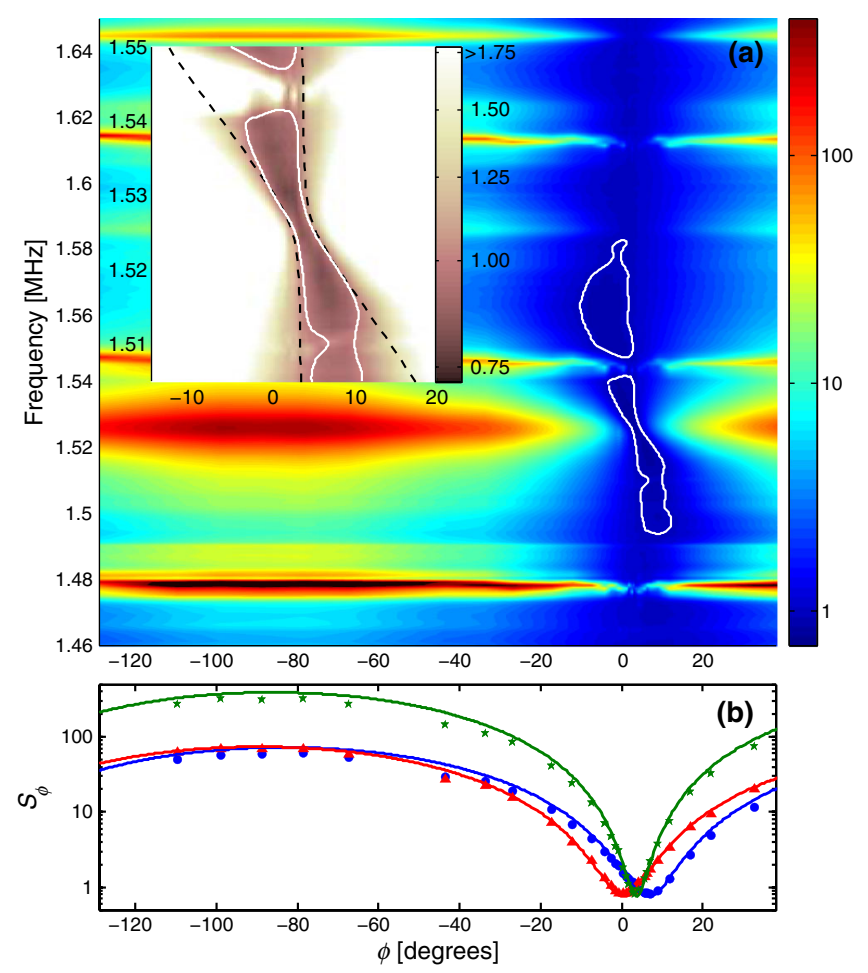

FIG. 3. Optical quadrature spectrum. (a) Color map of $S_{\phi}$. The white contour is at the shot-noise level, and the region inside this contour is squeezed. Several additional noise peaks are evident at frequencies away from the mechanical resonance, due to motion of thermally occupied modes of the support structure. The inset shows the squeezed region in more detail and also includes a theoretical prediction (dashed black curves) of the expected shotnoise contour. The experimental parameters are the same as in Fig. 2, except $\Delta / 2 \pi=-42 \mathrm{kHz}$. (b) Cuts through quadrature phase at three different frequencies $1.517 \mathrm{MHz}$ (blue circles), $1.526 \mathrm{MHz}$ (green stars), and $1.535 \mathrm{MHz}$ (red triangles), averaged over a $1-\mathrm{kHz}$ bandwidth, and corresponding to zero-freeparameter theoretical models (colored solid curves).

than 10 larger than the probe power, making the measurement slightly susceptible to the noise of the local oscillator (see the Appendix).

Homodyne detection also allows us to quantify the coherent amplification of optical quantum fluctuations in our measurement. In Fig. 3(b), we compare our data to a theoretical calculation based upon a Heisenberg-Langevin model. The agreement between the model and the data allows us to interpret the large noise spectral density $\left(S_{\phi} \sim 330\right)$ near the phase quadrature at $\phi= \pm 90^{\circ}$ as arising mainly from coherent amplification of quantum noise or so-called antisqueezing. This amplification persists despite the large imaginary component of the mechanical response, which has the potential to limit squeezing and add thermal noise. Note that the measured spectral densities are far in excess of that required to satisfy the Heisenberg uncertainty limit $\sqrt{S_{\phi}(\omega)} \sqrt{S_{\phi+\pi / 2}(\omega)} \gg 1$. In the Supplemental Material, we present the parameters and configuration that would be required to realize a minimum uncertainty state that saturates the Heisenberg bound [28].

In conclusion, we have experimentally demonstrated that an optomechanical system well into the RPSN-dominated regime is capable of creating squeezed light. The 1.7-dB strength of optomechanical squeezing we achieve is significantly larger than previous optomechanical realizations $[23,24]$. However, stronger squeezing has of course been realized with more developed techniques [15], and increasing efficiency and reducing thermal noise will be required to study the ultimate limits to deeply ponderomotively squeezed light. It will also be interesting to compare the passive squeezing achieved here to techniques that utilize optomechanically mediated quantum nondemolition measurements of the optical field and active feedback on the light $[29,30]$.

This material is based upon work supported by the National Science Foundation under Grant No. 1125844, by the ONR Young Investigator Program, and by the DARPA QuASAR Program. C.A.R. thanks the Clare Boothe Luce Foundation for support.

\section{APPENDIX: CALCULATION OF OPTICAL SPECTRA}

In this Appendix, we describe our solution to the Heisenberg-Langevin equations of motion for our optomechanical system. We then compute the expected output optical quadrature spectrum and the spectrum obtained from balanced homodyne detection and direct photodetection.

\section{Heisenberg-Langevin equations}

We begin with the following Hamiltonian $H=H_{0}+$ $H_{\kappa}+H_{\Gamma}$, where $H_{0}$ describes the intracavity coherent dynamics, $H_{\kappa}$ represents the coupling of the optical system to external fields, and $H_{\Gamma}$ represents the external thermal coupling to the mechanics [3-5,25,31]

$$
H_{0}=\hbar \omega_{m} c^{\dagger} c+\hbar \omega_{c} a^{\dagger} a+\hbar G Z_{\text {zp }}\left(c+c^{\dagger}\right) a^{\dagger} a,
$$

where $\omega_{m}$ is the mechanical resonance frequency, $c\left(c^{\dagger}\right)$ is the mechanical annihilation (creation) operator, $\omega_{c}$ is the optical resonance frequency, $a\left(a^{\dagger}\right)$ is the optical intracavity annihilation (creation) operator, $G$ is the optomechanical coupling constant, and $Z_{\mathrm{zp}}=\sqrt{\hbar / 2 m \omega_{m}}$ is the mechanical zero-point motion, with $m$ the mechanical resonator effective mass. We define a single-photon optomechanical coupling rate $g=G Z_{\text {zp }}$ and a dimensionless mechanical displacement operator $z=\left(c+c^{\dagger}\right)-\left\langle c+c^{\dagger}\right\rangle$. The Hamiltonian is linearized by assuming a large optical coherent-state amplitude compared to the vacuum level $a=[\bar{a}+d(t)] e^{i \omega_{L} t}$, where $\omega_{L}$ is the optical drive frequency, $\bar{a}=\langle a\rangle$ is the intracavity coherent-state amplitude, assumed to be real, and $d(t)$ is an operator 
containing the quantum and classical noise on the optical field. Terms of order $d^{2}$ are neglected. The linearized Hamiltonian that encapsulates the basic interaction is

$$
\begin{gathered}
H_{0}=\hbar \omega_{m} c^{\dagger} c+\hbar \omega_{c} a^{\dagger} a+\hbar G Z_{\mathrm{zp}}\left(c+c^{\dagger}\right) \bar{a}^{*} \bar{a}+H_{\mathrm{BS}} \\
+H_{\mathrm{TMS}} \\
H_{\mathrm{BS}}=\hbar G Z_{\mathrm{zp}}\left(\bar{a}^{*} c^{\dagger} d+\bar{a} c d^{\dagger}\right) \\
H_{\mathrm{TMS}}=\hbar G Z_{\mathrm{zp}}\left(\bar{a}^{*} c d+\bar{a} c^{\dagger} d^{\dagger}\right)
\end{gathered}
$$

The resulting linearized Hamiltonian contains both the beam splitter $\left(H_{\mathrm{BS}}\right)$ and the two-mode squeezing $\left(H_{\mathrm{TMS}}\right)$ Hamiltonians.

We solve the Heisenberg-Langevin equations of motion for this system in the frequency domain, using the Fourier transformation convention $f(\omega) \equiv \int_{-\infty}^{\infty} e^{l \omega t} f(t) d t$, $f^{\dagger}(\omega) \equiv \int_{-\infty}^{\infty} e^{i \omega t} f^{\dagger}(t) d t$, and $\left[f^{\dagger}(\omega)\right]^{\dagger}=f(-\omega)$. We assume thermally driven mechanical motion, with mechanical damping rate $\Gamma_{0}$ and initial thermal occupation $n_{\mathrm{th}}$. We include the effects of the additional optical damping beam in an orthogonal cavity mode by defining effective values for $\omega_{m}, \Gamma_{0}$, and $n_{\text {th }}$ for the motion of the mechanical resonator in the presence of the optomechanical damping, spring, and cooling induced by the damping beam [25]. The optomechanical effects of the signal beam are intrinsic in the equations of motion. The optical loss rate to the input port, output port, and internal loss are $\kappa_{L}, \kappa_{R}$, and $\kappa_{\text {int }}$, respectively, yielding a total cavity damping rate of $\kappa=$ $\kappa_{L}+\kappa_{R}+\kappa_{\text {int }}$. The external optical input fields consist of a coherent state, of frequency $\omega_{L}$, incident on the input port of the two-sided Fabry-Perot cavity, and vacuum incident on the output port. An effective detuning of the input signal field from the average value of the optomechanically shifted cavity resonance is given by $\Delta$. The optical output operator $a_{\text {out }}=\left[\bar{a}_{\text {out }}+d_{\text {out }}(t)\right] e^{i \omega_{L} t}$ is computed using the cavity input-output relations $\bar{a}_{\text {out }}=\sqrt{\kappa_{R}} \bar{a}$ and $d_{\text {out }}+$ $d_{\text {in }}=\sqrt{\kappa_{R}} d$, where $d_{\text {in }}$ is the noise operator representing the vacuum field incident on the output port [32].

\section{Optical output spectrum}

The quadrature output operator is defined as $X_{\phi}(\omega)=$ $a_{\text {out }}(\omega) e^{i \phi}+a_{\text {out }}^{\dagger}(\omega) e^{-i \phi}$, where $\phi$ is the quadrature phase angle. Because we have assumed $\bar{a}$ to be real, the inputoutput relation indicates that $\bar{a}_{\text {out }}$ is also real, and $\phi=0$ $(\phi=\pi / 2)$ corresponds to the amplitude (phase) quadrature. The symmetrized power spectrum of the quadrature operator is $S_{X X}(\omega)$ :

$$
\begin{aligned}
S_{X X}(\omega) & =\left\langle X_{\phi}(-\omega) X_{\phi}(\omega)\right\rangle_{s} \\
& =\frac{1}{2}\left[\left\langle X_{\phi}(-\omega) X_{\phi}(\omega)\right\rangle+\left\langle X_{\phi}(\omega) X_{\phi}(-\omega)\right\rangle\right] \\
& =A_{\zeta \zeta}(\omega)+A_{z z}(\omega)+A_{\zeta z}(\omega) .
\end{aligned}
$$

The spectrum consists of three terms. $A_{\zeta \zeta}$ is the shot noise on the output. $A_{z z}$ represents the noise imprinted by the actual mechanical motion. The cross term $A_{\zeta z}$ contains the correlations between shot noise and motion driven by radiation pressure from the shot noise. This term is responsible for any squeezing:

$$
\begin{aligned}
A_{z z}(\omega)= & \kappa_{R}|\bar{a}|^{2} g^{2}\left[\left|\chi_{c}(\omega)\right|^{2}+\left|\chi_{c}(-\omega)\right|^{2}-\chi_{c}(\omega) \chi_{c}(-\omega) e^{2 i \phi}-\chi_{c}^{*}(\omega) \chi_{c}^{*}(-\omega) e^{-2 i \phi}\right]\langle z(-\omega) z(\omega)\rangle_{s}, \\
A_{\zeta z}(\omega)= & i \sqrt{\kappa_{R}} \bar{a} g\left\{\left[-\chi_{c}(-\omega) e^{2 i \phi}+\chi_{c}^{*}(\omega)\right]\langle z(-\omega) \zeta(\omega)\rangle_{s}+\left[-\chi_{c}(\omega) e^{2 i \phi}+\chi_{c}^{*}(-\omega)\right]\langle\zeta(-\omega) z(\omega)\rangle_{s}\right. \\
& \left.+\left[\chi_{c}^{*}(\omega) e^{-2 i \phi}-\chi_{c}(-\omega)\right]\left\langle z(-\omega) \zeta^{\dagger}(\omega)\right\rangle_{s}+\left[\chi_{c}^{*}(-\omega) e^{-2 i \phi}-\chi_{c}(\omega)\right]\left\langle\zeta^{\dagger}(-\omega) z(\omega)\right\rangle_{s}\right\}, \\
A_{\zeta \zeta}(\omega)= & \left\langle\zeta^{\dagger}(-\omega) \zeta(\omega)\right\rangle_{s}+\left\langle\zeta(-\omega) \zeta^{\dagger}(\omega)\right\rangle_{s}=1 .
\end{aligned}
$$

The output shot-noise operator is $\zeta(\omega)=\chi_{c}(\omega) \sqrt{\kappa_{L} \kappa_{R}} \xi_{L}(\omega)+\chi_{c}(\omega) \sqrt{\kappa_{\text {int }} \kappa_{R}} \xi_{\text {int }}(\omega)+\left[\chi_{c}(\omega) \kappa_{R}-1\right] \xi_{R}(\omega) . \xi_{L}, \xi_{R}$, and $\xi_{\text {int }}$ are the Langevin vacuum noise operators for the input port, output port, and internal loss of the cavity, respectively:

$$
\begin{aligned}
\langle z(-\omega) z(\omega)\rangle_{s} & =\frac{1}{|\mathcal{N}(\omega)|^{2}}\left[\Gamma_{0}\left(\frac{n_{\mathrm{th}}+1 / 2}{\left|\chi_{m}(\omega)\right|^{2}}+\frac{n_{\mathrm{th}}+1 / 2}{\left|\chi_{m}(-\omega)\right|^{2}}\right)+2 \omega_{m}^{2} g^{2} \kappa|\bar{a}|^{2}\left[\left|\chi_{c}(\omega)\right|^{2}+\left|\chi_{c}(-\omega)\right|^{2}\right]\right] \\
\langle z(-\omega) \zeta(\omega)\rangle_{s} & =\frac{-\omega_{m} \bar{a} g \sqrt{\kappa_{R}}}{\mathcal{N}(-\omega)} \chi_{c}(\omega), \quad\langle\zeta(-\omega) z(\omega)\rangle_{s}=\frac{-\omega_{m} \bar{a} g \sqrt{\kappa_{R}}}{\mathcal{N}(\omega)} \chi_{c}(-\omega), \quad\left\langle\zeta^{\dagger}(-\omega) \zeta(\omega)\right\rangle_{s}=\left\langle\zeta(-\omega) \zeta^{\dagger}(\omega)\right\rangle_{s}=\frac{1}{2}
\end{aligned}
$$

We have introduced the following notation. The cavity susceptibility is $\chi_{c}(\omega)=[\kappa / 2-i(\Delta+\omega)]^{-1}$. The mechanical susceptibility is $\chi_{m}(\omega)=\left[\Gamma_{0} / 2-i\left(\omega-\omega_{m}\right)\right]^{-1}$. The optomechanical damping and spring effects are encompassed in $\mathcal{N}(\omega)=\left[\chi_{m}(\omega) \chi_{m}^{*}(-\omega)\right]^{-1}-i 2 \omega_{m} g^{2}|\bar{a}|^{2} \times$ $\left[\chi_{c}(\omega)-\chi_{c}^{*}(-\omega)\right]$. We also assume the mechanical thermal and optical vacuum baths are uncorrelated at different times $\left\langle\xi\left(-\omega^{\prime}\right) \xi(\omega)\right\rangle=\delta\left(\omega-\omega^{\prime}\right)$, for noise operator $\xi$, and we assume integration over $\omega^{\prime}$ for physically relevant quantities.

Any loss in the optical detection system, including propagation losses between the cavity and detector, imperfect mode matching to the homodyne detector, or finite photodetector conversion efficiency, can be modeled by a single effective loss port with fractional loss $\epsilon_{\text {ext }}$. The loss port attenuates the signal reaching the detector 


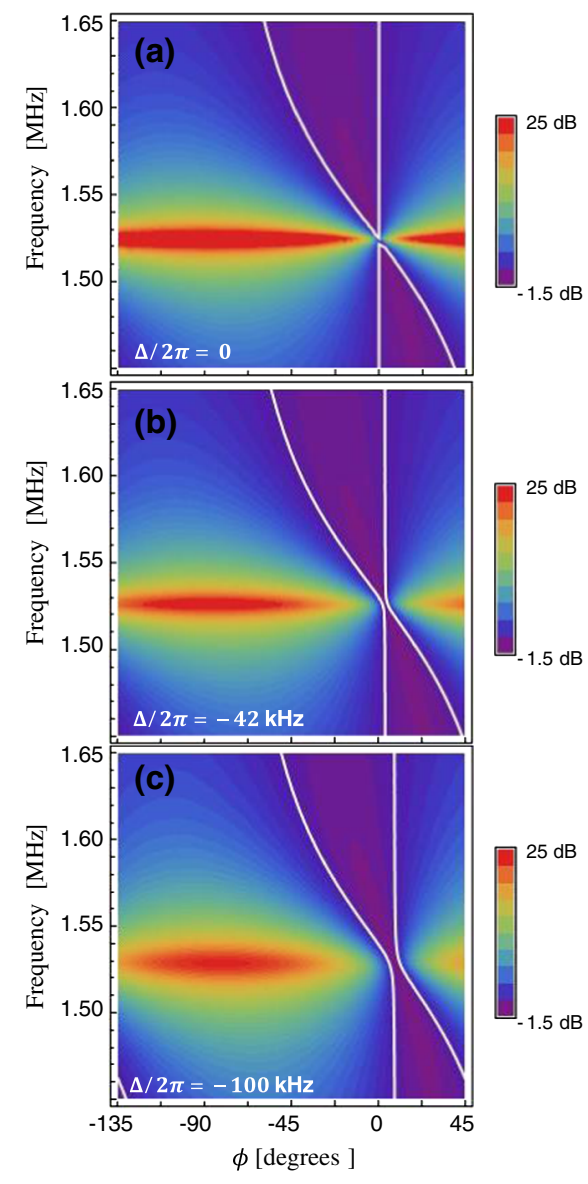

FIG. 4. Calculated homodyne spectrum for finite signal-beam detuning. Homodyne transmission spectra $S_{\phi}$ are calculated for three signal-beam-cavity detunings: (a) $\Delta / 2 \pi=0$, (b) $\Delta / 2 \pi=-42 \mathrm{kHz}$, and (c) $\Delta / 2 \pi=-100 \mathrm{kHz}$. The other parameters used are $g / 2 \pi=33 \mathrm{~Hz}, m=6.75 \times 10^{-12} \mathrm{~kg}$, $\omega_{m}^{\text {eff }} / 2 \pi=1.5243 \mathrm{MHz}, \quad \Gamma_{0}^{\text {eff }} / 2 \pi=2560 \mathrm{~Hz}, \quad T^{\text {eff }}=$ $3.8 \times 10^{-4} \mathrm{~K}, \epsilon_{\mathrm{ext}}=0.55, \kappa / 2 \pi=1.7 \mathrm{MHz}, \kappa_{R}=0.6 \kappa, \bar{N}=$ $1.1 \times 10^{8}$, and $\epsilon_{\text {ext }}\left|\bar{a}_{\text {out }}\right|^{2} /\left|\bar{a}_{\mathrm{LO}}\right|^{2}=0.1$. The parameters of (b) match the parameters of the measured spectrum presented in Fig. 3. With those parameters, the mechanical damping from the signal beam is $6 \mathrm{kHz}$. Calculated spectra are displayed on a logarithmic scale. The region between the white $0-\mathrm{dB}$ contours is squeezed.

$\bar{a}_{\text {out }} \rightarrow \sqrt{\epsilon_{\text {ext }}} \bar{a}_{\text {out }}$ and injects vacuum noise, leading an effective quadrature spectrum of $S_{X X}(\omega) \rightarrow \epsilon_{\mathrm{ext}} S_{X X}(\omega)+$ $\left(1-\epsilon_{\mathrm{ext}}\right)$.

The homodyne detection consists of combining a strong optical local oscillator with the output from the cavity on a beam splitter. Both outputs of the beam splitter are recorded on photodetectors, and the two photocurrents are subtracted. Assuming an equal splitting on the beam splitter, the subtracted photocurrent signal is proportional to $\left(\bar{a}_{\text {out }} \bar{a}_{\mathrm{LO}} e^{i \phi}-\bar{a}_{\text {out }} \bar{a}_{\mathrm{LO}} e^{-i \phi}\right)+\bar{a}_{\text {out }}\left(d_{\mathrm{LO}}+d_{\mathrm{LO}}^{\dagger}\right)+$ $\bar{a}_{\mathrm{LO}}\left(d_{\mathrm{out}} e^{i \phi}+d_{\text {out }}^{\dagger} e^{-i \phi}\right)$, where the annihilation operator of the local oscillator is $a_{\mathrm{LO}}=\left[\bar{a}_{\mathrm{LO}}+d_{\mathrm{LO}}(t)\right] e^{i \omega_{L} t+\phi}$, and we have neglected terms of order $d^{2}$. The third term is proportional to $X_{\phi}$. The second term, which represents the local oscillator vacuum noise beating against the coherent portion of the cavity output field, is negligible when $\bar{a}_{\mathrm{LO}} \gg$ $\bar{a}_{\text {out }}$ and is typically ignored. However, in the homodyne detection system described in the main text, we are limited to a local oscillator power that is less than 10 times larger than the signal-beam power in order to ensure that the photodetectors remain in their linear range. In this case, the local oscillator noise term must be included to quantitatively model the homodyne data. The one-sided, symmetrized, shot-noise-normalized spectra $S_{\phi}(\omega)$ in the main text are then given by

$$
S_{\phi}(\omega)=\frac{2\left\{\left|\bar{a}_{\mathrm{LO}}\right|^{2}\left[\epsilon_{\mathrm{ext}} S_{X X}(\omega)+\left(1-\epsilon_{\mathrm{ext}}\right)\right]+\epsilon_{\mathrm{ext}}\left|\bar{a}_{\mathrm{out}}\right|^{2}\right\}}{2\left(\left|\bar{a}_{\mathrm{LO}}\right|^{2}+\epsilon_{\mathrm{ext}}\left|\bar{a}_{\mathrm{out}}\right|^{2}\right)} .
$$

Using this full expression, one sees that the level of perceived squeezing is reduced by the additional uncorrelated noise floor of the local oscillator.

The one-sided, symmetrized, shot-noise-normalized, direct photodetection spectrum, discussed in the main text, requires $S_{X X}(\omega)$ to be evaluated at $\phi=0$ :

$$
S_{I}(\omega)=\left.\epsilon_{\mathrm{ext}} S_{X X}(\omega)\right|_{\phi=0}+\left(1-\epsilon_{\mathrm{ext}}\right) .
$$

Several calculated spectra of $S_{\phi}$ in Fig. 4 illustrate the effects of the signal-beam detuning $\Delta$. Three different values of $\Delta$ are displayed, and the other parameters are chosen to match the experimental data of the balanced homodyne experiment. Two trends are evident as $\Delta$ is varied. First, the entire spectrum is rotated by the cavity filtering by $\tan ^{-1}(2 \Delta / \kappa)$. This rotation is most evident by focusing on the white 0 - $\mathrm{dB}$ contour, which is shifted away from $\phi=0$ for increasing detuning. Second, the optomechanical damping from the signal becomes significant for nonzero detuning, consequently broadening the features. This broadening is evident near the phase quadrature, which when $\Delta \sim 0$ is proportional to the actual mechanical motion. The area between the $0-\mathrm{dB}$ shot-noise contours also becomes noticeably wider near the amplitude quadrature and near $\omega_{m}$ as $\Delta$ is increased.

[1] J. D. Teufel, T. Donner, Dale Li, J.W. Harlow, M. S. Allman, K. Cicak, A. J. Sirois, J. D. Whittaker, K. W. Lehnert, and R.W. Simmonds, Sideband Cooling of Micromechanical Motion to the Quantum Ground State, Nature (London) 475, 359 (2011).

[2] Jasper Chan, T. P. Mayer Alegre, Amir H. Safavi-Naeini, Jeff T. Hill, Alex Krause, Simon Gröblacher, Markus Aspelmeyer, and Oskar Painter, Laser Cooling of a Nanomechanical Oscillator into Its Quantum Ground State, Nature (London) 478, 89 (2011).

[3] T. P. Purdy, R. W. Peterson, and C. A. Regal, Observation of Radiation Pressure Shot Noise on a Macroscopic Object, Science 339, 801 (2013). 
[4] C. Fabre, M. Pinard, S. Bourzeix, A. Heidmann, E. Giacobino, and S. Reynaud, Quantum-Noise Reduction Using a Cavity with a Movable Mirror, Phys. Rev. A 49, 1337 (1994).

[5] S. Mancini and P. Tombesi, Quantum Noise Reduction by Radiation Pressure, Phys. Rev. A 49, 4055 (1994).

[6] V.B. Braginsky and A. B. Manukin, Ponderomotive Effects of Electromagnetic Radiation, Sov. Phys. JETP 25, 653 (1967) [http://www.jetp.ac.ru/cgi-bin/e/index/e/ 25/4/p653?a=list].

[7] V. B. Braginsky and F. Y. Khalili, Quantum Measurement (Cambridge University Press, Cambridge, England, 1992).

[8] Carlton M. Caves, Quantum-Mechanical Noise in an Interferometer, Phys. Rev. D 23, 1693 (1981).

[9] W. G. Unruh, in Quantum Optics, Experimental Gravitation, and Measurement Theory, edited by $\mathrm{P}$. Meystre and M.O. Scully (Plenum, New York, 1982), p. 647.

[10] M. T. Jaekel and S. Reynaud, Quantum Limits in Interferometric Measurements, Europhys. Lett. 13, 301 (1990).

[11] H. J. Kimble, Yuri Levin, Andrey B. Matsko, Kip S. Thorne, and Sergey P. Vyatchanin, Conversion of Conventional Gravitational-Wave Interferometers into Quantum Nondemolition Interferometers by Modifying Their Input and/or Output Optics, Phys. Rev. D 65, 022002 (2001).

[12] R. E. Slusher, L. W. Hollberg, B. Yurke, J. C. Mertz, and J. F. Valley, Observation of Squeezed States Generated by Four-Wave Mixing in an Optical Cavity, Phys. Rev. Lett. 55, 2409 (1985).

[13] R. M. Shelby, M. D. Levenson, S. H. Perlmutter, R. G. DeVoe, and D.F. Walls, Broad-Band Parametric Deamplification of Quantum Noise in an Optical Fiber, Phys. Rev. Lett. 57, 691 (1986).

[14] Ling-An Wu, H.J. Kimble, J. L. Hall, and Huifa Wu, Generation of Squeezed States by Parametric Down Conversion, Phys. Rev. Lett. 57, 2520 (1986).

[15] Tobias Eberle, Sebastian Steinlechner, Jöran Bauchrowitz, Vitus Händchen, Henning Vahlbruch, Moritz Mehmet, Helge Müller-Ebhardt, and Roman Schnabel, Quantum Enhancement of the Zero-Area Sagnac Interferometer Topology for Gravitational Wave Detection, Phys. Rev. Lett. 104, 251102 (2010).

[16] R. Schnabel et al. (LIGO Scientific Collaboration), A Gravitational Wave Observatory Operating beyond the Quantum Shot-Noise Limit, Nat. Phys. 7, 962 (2011).

[17] Michael A. Taylor, Jiri Janousek, Vincent Daria, Joachim Knittel, Boris Hage, Hans-A. Bachor, and Warwick P. Bowen, Biological Measurement beyond the Quantum Limit, Nat. Photonics 7, 229 (2013).

[18] M. A. Castellanos-Beltran, K. D. Irwin, G. C. Hilton, L. R. Vale, and K. W. Lehnert, Amplification and Squeezing of
Quantum Noise with a Tunable Josephson Metamaterial, Nat. Phys. 4, 929 (2008).

[19] L. Hilico, J. M. Courty, C. Fabre, E. Giacobino, I. Abram, and J.L. Oudar, Squeezing with $\chi^{(3)}$ Materials, Appl. Phys. B 55, 202 (1992).

[20] Thomas Corbitt, Yanbei Chen, Farid Khalili, David Ottaway, Sergey Vyatchanin, Stan Whitcomb, and Nergis Mavalvala, Squeezed-State Source Using Radiation-Pressure-Induced Rigidity, Phys. Rev. A 73, 023801 (2006).

[21] A. Heidmann, Y. Hadjar, and M. Pinard, Quantum Nondemolition Measurement by Optomechanical Coupling, Appl. Phys. B 64, 173 (1997).

[22] A. Dorsel, J. D. McCullen, P. Meystre, E. Vignes, and H. Walther, Optical Bistability and Mirror Confinement Induced by Radiation Pressure, Phys. Rev. Lett. 51, 1550 (1983).

[23] Daniel W. C. Brooks, Thierry Botter, Sydney Schreppler, Thomas P. Purdy, Nathan Brahms, and Dan M. StamperKurn, Non-classical Light Generated by Quantum-NoiseDriven Cavity Optomechanics, Nature (London) 488, 476 (2012).

[24] A. H. Safavi-Naeini, S. Gröblacher, J. T. Hill, J. Chan, M. Aspelmeyer, and O. Painter, Squeezed Light from a Silicon Micromechanical Resonator, Nature (London) 500, 185 (2013).

[25] F. Marquardt, J. P. Chen, A. A. Clerk, and S. M. Girvin, Quantum Theory of Cavity-Assisted Sideband Cooling of Mechanical Motion, Phys. Rev. Lett. 99, 093902 (2007).

[26] T. P. Purdy, R. W. Peterson, P.-L. Yu, and C. A. Regal, Cavity Optomechanics with $\mathrm{Si}_{3} \mathrm{~N}_{4}$ Membranes at Cryogenic Temperatures, New J. Phys. 14, 115021 (2012).

[27] J.D. Thompson, B. M. Zwickl, A. M. Jayich, Florian Marquardt, S. M. Girvin, and J.G.E. Harris, Strong Dispersive Coupling of a High-Finesse Cavity to a Micromechanical Membrane, Nature (London) 452, 72 (2008).

[28] See Supplemental Material at http://link.aps.org/ supplemental/10.1103/PhysRevX.3.031012 for details about experimental methods, calibrations, and additional calculations.

[29] H. M. Wiseman and G.J. Milburn, Squeezing via Feedback, Phys. Rev. A 49, 1350 (1994).

[30] S. Mancini and H.M. Wiseman, Optomechanical Tailoring of Quantum Fluctuations, J. Opt. B 2, 260 (2000).

[31] Thierry Botter, Daniel W.C. Brooks, Nathan Brahms, Sydney Schreppler, and Dan M. Stamper-Kurn, Linear Amplifier Model for Optomechanical Systems, Phys. Rev. A 85, 013812 (2012).

[32] D. F. Walls and G. J. Milburn, Quantum Optics (SpringerVerlag, Berlin, 2008), 2nd ed. 